\title{
Endotaxial growth mechanisms of Sn Quantum Dots in Si matrix
}

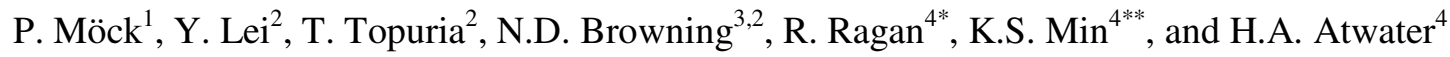 \\ ${ }^{1}$ Department of Physics, Portland State University, P.O. Box 751, Portland, OR 97207-0751, pmoeck@pdx.edu \\ ${ }^{2}$ Department of Physics, University of Illinois at Chicago, 845 W. Taylor Street, Chicago, IL 60607-7059 \\ ${ }^{3}$ Department of Chemical Engineering and Materials Science, University of California at Davis, One Shields \\ Avenue, Davis, CA 95616; and National Center for Electron Microscopy, MS 72-150, Lawrence Berkeley National \\ Laboratory, Berkeley, CA 94720 \\ ${ }^{4}$ Thomas J. Watson Laboratory of Applied Physics, California Institute of Technology, MS 128-95, Pasadena, CA \\ 91125 * now at: Hewlett-Packard Laboratories M/S 1123, 1501 Page Mill Rd., Palo Alto, CA 94304, ** now at: \\ Intel Corporation, California Technology and Manufacturing, MS RNB-2-35, 2200 Mission College Blvd., Santa \\ Clara, CA 95052-8119
}

\begin{abstract}
Two distinct mechanisms for the endotaxial growth of quantum dots in the $\mathrm{Sn} / \mathrm{Si}$ system were observed by means of analytical transmission electron microcopy. Both mechanisms operate simultaneously during temperature and growth rate modulated molecular beam epitaxy combined with ex situ thermal treatments. One of the mechanisms involves the creation of voids in $\mathrm{Si}$, which are subsequently filled by $\mathrm{Sn}$, resulting in quantum dots that consist of pure $\alpha$-Sn. The other mechanism involves phase separation and leads to substitutional solid solution quantum dots with a higher Sn content than the predecessor quantum well structures possess. In both cases, the resultant quantum dots possess the diamond structure and the shape of a tetrakaidecahedron. $(\mathrm{Sn}, \mathrm{Si})$ precipitates that are several times larger than the typical $(\mathrm{Sn}, \mathrm{Si})$ quantum dot possess an essentially octahedral shape.
\end{abstract}

\section{INTRODUCTION}

Self-assembled semiconductor quantum dots (QDs) are expected to lead to "paradigm changes in semiconductor physics" [1]. For semiconductor opto-electronic devices, the QD size must be of the order of magnitude of the exciton Bohr radius. The energy band gap of the QDs must be smaller than that of the surrounding semiconductor matrix. No structural defects such as dislocations, which lead to non-radiative recombination, are allowed to exist in the QDs [2].

As $\alpha$-Sn is a direct, $0.08 \mathrm{eV}$, band gap semiconductor and substitutional $\mathrm{Sn}_{\mathrm{x}} \mathrm{Si}_{1-\mathrm{x}}$ solution are predicted to possess direct band gaps for $0.9<\mathrm{x}<1$ [3], QDs in a Si matrix consisting of pure $\alpha$-Sn or substitutional ( $\mathrm{Sn}, \mathrm{Si}$ ) solutions with a sufficiently high Sn content have potential applications as direct band-gap material for cheap and effective optoelectronic and thermo-photovoltaic devices. There are, however, at room temperature a $41.8 \%$ bulk unit cell volume mismatch between $\alpha-\mathrm{Sn}$ and $\mathrm{Si}$ and an equilibrium solid solubility of $\mathrm{Sn}$ in $\mathrm{Si}$ of only $0.12 \%$. This restricts the growth of pseudomorph $\mathrm{Sn}_{\mathrm{x}} \mathrm{Si}_{1-\mathrm{x}}$ layers on $\mathrm{Si}$ by molecular beam epitaxy (MBE) [4-7] to a Sn content of about $10 \%$ and a thickness of the order of magnitude $10 \mathrm{~nm}$.

At growth temperatures in the range 220 to $295{ }^{\circ} \mathrm{C}$, pseudomorph $\mathrm{Sn}_{\mathrm{x}} \mathrm{Si}_{1-\mathrm{x}}$ layers with up to $5 \% \mathrm{Sn}$ content have been grown with film thicknesses up to $170 \mathrm{~nm}$. Thermal treatments of these layers at temperatures above $500{ }^{\circ} \mathrm{C}$ for 1 hour led to the formation of $\alpha$-Sn precipitates, $\beta$-Sn precipitates, precipitates that consisted of both $\alpha$-Sn and $\beta$ Sn, and misfit dislocations [5-7]. While these $\alpha$-Sn precipitates may be considered to constitute QDs in this material system (according to the requirements given above [2]), the simultaneously present misfit dislocations are clearly undesirable for device applications.

Alternatively, temperature and growth rate modulated $\mathrm{MBE}[8,9]$ produces $\mathrm{Sn}_{\mathrm{x}} \mathrm{Si}_{1-x} / \mathrm{Si}$ superlattices with essentially pseudomorph $\mathrm{Sn}_{\mathrm{x}} \mathrm{Si}_{1-\mathrm{x}}$ substitutional solutions having $\mathrm{Sn}$ compositions in the range of $\mathrm{x}=0.02$ to 0.05 and film thicknesses ranging from 1 to $2 \mathrm{~nm}$. Typical growth procedures $[8,9]$ are as follows.

The growth temperatures of the $\mathrm{Sn}_{x} \mathrm{Si}_{1-\mathrm{x}}$ layers range from 140 to $170{ }^{\circ} \mathrm{C}$ and the growth rate is $0.02 \mathrm{~nm}$ per second. To suppress segregation of $\mathrm{Sn}$ to the surface during growth, the $\mathrm{Sn}_{\mathrm{x}} \mathrm{Si}_{1-\mathrm{x}}$ layers are overgrown with 4 to $6 \mathrm{~nm}$ of $\mathrm{Si}$ at the $\mathrm{Sn}_{x} \mathrm{Si}_{1-x}$ growth temperature and at growth rates ranging from 0.01 to $0.03 \mathrm{~nm}$ per second. The temperature is then raised to $550{ }^{\circ} \mathrm{C}$ and a $\mathrm{Si}$ capping layer with a thickness of the order of magnitude $100 \mathrm{~nm}$ is grown at a rate of $0.05 \mathrm{~nm}$ per second. By the time this growth sequence has been completed, the $\mathrm{Sn}_{\mathrm{x}} \mathrm{Si}_{1-\mathrm{x}}$ layer has experienced an in situ thermal treatment at $550{ }^{\circ} \mathrm{C}$ for a time of the order of magnitude 30 minutes. For the growth of $\mathrm{Sn}_{\mathrm{x}} \mathrm{Si}_{1-\mathrm{x}} / \mathrm{Si}$ multilayer structures, the whole growth sequence is repeated several times, effectively resulting in an 
in situ thermal treatment for the first $\mathrm{Sn}_{\mathrm{x}} \mathrm{Si}_{1-\mathrm{x}}$ layer at $550{ }^{\circ} \mathrm{C}$ for a time on the order of magnitude a few hours. In addition to this in situ thermal treatment, ex situ anneals at temperatures between 550 and $900{ }^{\circ} \mathrm{C}$ are performed for 30 minutes. The summarily effect of the epitaxial growth and these thermal treatments is the crystallographically oriented precipitation of Sn within the Si matrix. Precipitation process such as this are broadly described as endotaxy (from the Greek, literally meaning arrangement within).

There is an unexplained peculiarity in the plots of average QD precipitate sizes versus ex-situ annealing times, e.g. Fig. 1a, subsequent to temperature and growth rate modulated MBE [9]. Employing analytical transmission electron microscopy in both the parallel illumination and scanning probe mode, we will address this issue in the main part of this paper. A minor issue to be addressed at the end of this paper is the shape of larger $(\mathrm{Sn}, \mathrm{Si})$ precipitates.

Thermodynamics driven structural transformations may occur in both $\alpha-S n$ and $\mathrm{Sn}_{\mathrm{y}} \mathrm{Si}_{1-\mathrm{y}}(\mathrm{y}>\mathrm{x})$ QDs. As one can easily estimate, there is an essentially hydrostatic pressure of the order of magnitude $10 \mathrm{GPa}$ on these entities with a corresponding excess Gibbs free energy that significantly exceeds the thermal energy of the atoms in the QDs [10]. This excess Gibbs free energy may be reduced by structural prototype transformations from the diamond structure into either the $\beta$-Sn structure or into atomically ordered Sn-Si compounds (over a long enough time, possibly even at room temperature [11]). Here we would like to mention only that we actually did observe in temperature and growth rate modulated MBE grown $\mathrm{Sn} / \mathrm{Si}$ quantum dot samples $\beta$-Sn precipitates [10] and precipitates with showed 1/2 $\{110\}$ superlattice spots in their [001] diffraction pattern [12] (besides $\alpha$-Sn and $\mathrm{Sn}$ rich $\mathrm{Sn}_{\mathrm{y}} \mathrm{Si}_{1-\mathrm{y}}$ QD precipitates).

Finally, we would like to remark that the equilibrium shape of a void in Si has been determined experimentally [13] to be a tetrakaidecahedron, Fig. 1b. This shape is determined by $\{111\}$, i.e. octahedron, and $\{100\}$, i.e. cube, planes. $\mathrm{A}=\mathrm{t} / \mathrm{a}$ is a shape parameter, Fig. $1 \mathrm{~b}$; for $\mathrm{A}=0$ the shape is an octahedron and $\mathrm{A}=2 / 3$ corresponds to a cube.

\section{EXPERIMENTAL DETAILS}

Three sets of pairs of multilayer samples (one with and one without an additional ex-situ anneal for 30 minutes at $800{ }^{\circ} \mathrm{C}$ ) with four $\mathrm{Sn}_{x} \mathrm{Si}_{1-x} / \mathrm{Si}$ layers and substitutional $\mathrm{Sn}$ contents of nominally $2 \%, 5 \%$, and $10 \%$ in each of the $\mathrm{Sn}_{\mathrm{x}} \mathrm{Si}_{1-\mathrm{x}}$ layers were grown by temperature and growth rate modulated MBE [8,9], stored at room temperature for a few years, and eventually selected for our transmission electron microcopy (TEM) investigations.

Our structural analyses employed analytical TEM in both the parallel illumination and scanning probe mode using both a JEOL JEM-2010F Schottky field emission STEM/TEM and a JEOL JEM-3010 TEM at the University of Illinois at Chicago. Parallel illumination TEM utilized conventional diffraction contrast (CTEM), selected area electron diffraction, and high-resolution phase contrast (HRTEM) imaging. Atomic resolution Z-contrast imaging in the scanning probe mode (STEM) proved to be especially useful for our investigations as the effects of strain fields in and around QDs and interference effects such as the formation of moiré fringe due to double diffraction are negligible [12]. In addition, the intensity in Z-contrast images is roughly proportional to the square of the atomic number $(Z)$ of the scattering atoms. For precipitated $\mathrm{Sn}(Z=50)$ in $\mathrm{Si}(Z=14)$, an image contrast as high as about $\left(50^{2} / 14^{2}\right)-1$ is possible, which allows for a direct and intuitive interpretation of such Z-contrast images in terms of a distribution map of Sn. TEM specimen preparation followed standard procedures involving mechanical grinding and ion milling to electron transparency.

\section{RESULTS AND DISCUSSIONS}

Earlier plan view TEM investigations of $\mathrm{Sn}_{\mathrm{x}} \mathrm{Si}_{1-\mathrm{x}}$ layers annealed at $650{ }^{\circ} \mathrm{C}$ at the California Institute of Technology revealed an initially rapid increase of the average QD volume $\left.(<\mathrm{r}\rangle^{3}\right)$ with time $(\mathrm{t})$ for the first 2.2 hours of the anneal, Fig. 1a, [9]. After this time, the function $\left\langle>^{3}(t)\right.$ showed the typical linear behavior that is expected for precipitate coarsening by volume diffusion [14]. Non-linearities of $\langle r\rangle^{3}$ with time are commonly attributed to diffusion shortcuts such as dislocations, stacking faults, grain boundaries, and other common lattice defects [14].

Our TEM/STEM investigations of similar samples in plan view and cross section, Figs. 2a-c, however, showed that these structures are essentially free of the common lattice defects, mentioned above [12]. The lower magnification cross section images of Figs. 2a,b show that there are small precipitates of Sn within the Si matrix quite far away from the spatial positions of the $\mathrm{Sn}_{\mathrm{x}} \mathrm{Si}_{1-\mathrm{x}}$ layers. Such $\mathrm{Sn}$ precipitates were found in both the ex-situ annealed and the as grown samples, which only had in situ thermal treatments. Higher magnification Z-contrast STEM images such as Fig. 3a show directly that these Sn precipitates are voids within the Si matrix that are partially filled with Sn. In this (Fig. 3a) image one can directly discern that Sn (which appears brighter due to its larger Z) lines the interface between the void and the Si matrix. As mentioned above, the equilibrium shape of a void in Si is a tetrakaidecahedron, Fig. 1b, [13]. The applications of Neumann's and Curie's symmetry principles [15] to the 
determination of the shape of small $\alpha-\mathrm{Sn}$ and $\mathrm{Sn}_{\mathrm{y}} \mathrm{Si}_{1-\mathrm{y}}$ precipitates in a $\mathrm{Si}$ matrix shows that this can be a tetrakaidecahedron as well $[10,16]$. Filling an equilibrium shape void in Si by precipitation of Sn should, therefore, be an energetically favorable process.

Additional evidence for the existence of voids in Si that are partly filled with $\mathrm{Sn}$ has been gathered by quantitative electron energy loss spectroscopy (EELS) [17], Fig. 3b, and will be presented elsewhere [18]. The filling up of partially $\mathrm{Sn}$ filled voids in Si by more $\mathrm{Sn}$ as a result of diffusion (into a diffusion shortcut) due to a thermal treatment at moderate parameters $\left(300{ }^{\circ} \mathrm{C}\right.$ for approximately three hours) was directly (in situ) observed under the electron microscope, Figs. 4a,b. More or less well formed $\alpha$-Sn QDs, Fig. 4b, resulted as $\alpha$-Sn and Si both possess the diamond structure. We consider this latter in situ observation as most direct proof of our void-filling hypothesis, proposed above.

This mechanism also provides a straightforward explanation for the initially rapid increase of the average QD precipitate volume with annealing time, $<\mathrm{r}>^{3}(\mathrm{t})$, in Fig. 1a. The creation of voids in $\mathrm{Si}$ and their subsequent filling with Sn emerges, therefore, as the first of the two mechanisms by which quantum dots grow endotaxially in the $\mathrm{Sn} / \mathrm{Si}$ system.
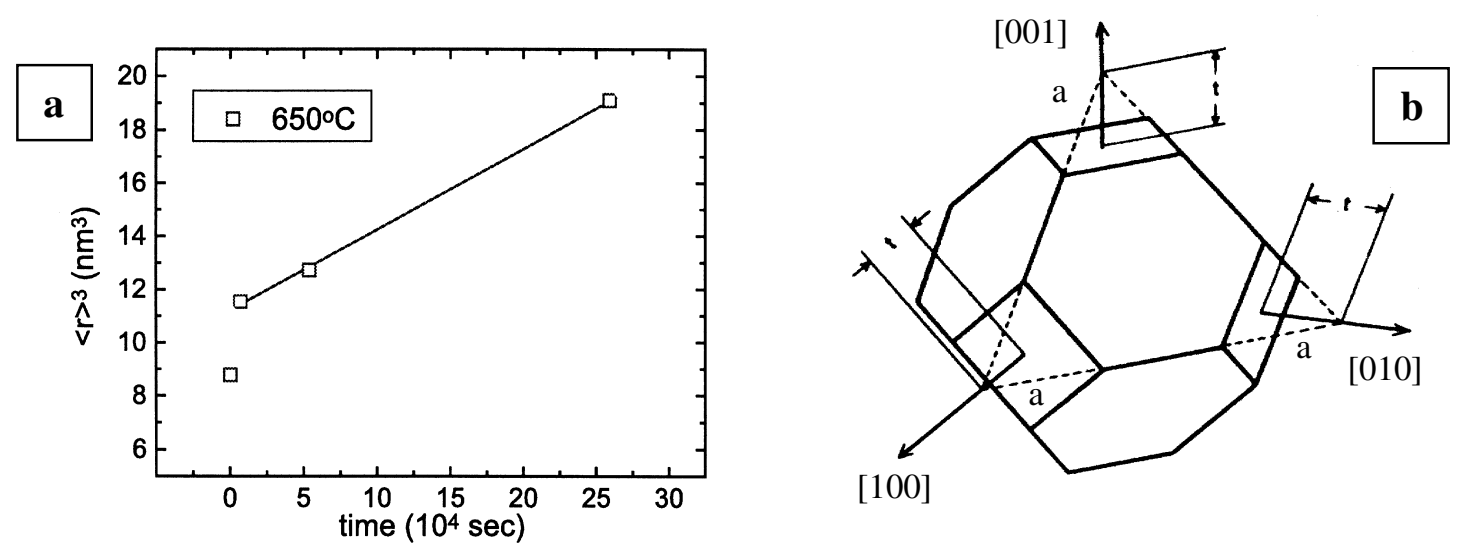

Figure 1. (a) Results of earlier ex situ annealing experiments at $650{ }^{\circ} \mathrm{C}$, after ref. [9]. The plot of the average QD precipitate size $\left(\langle r\rangle^{3}\right)$ versus the annealing time $(\mathrm{t})$ shows an initial rapid increase of the $\langle\mathrm{r}\rangle^{3}(\mathrm{t})$ function which later on shows the typical linear relationship that is expected when the precipitate coarsening is governed by volume diffusion. (b) Sketch of a tetrakaidecahedron, modified after ref. [15] (with that author's permission).
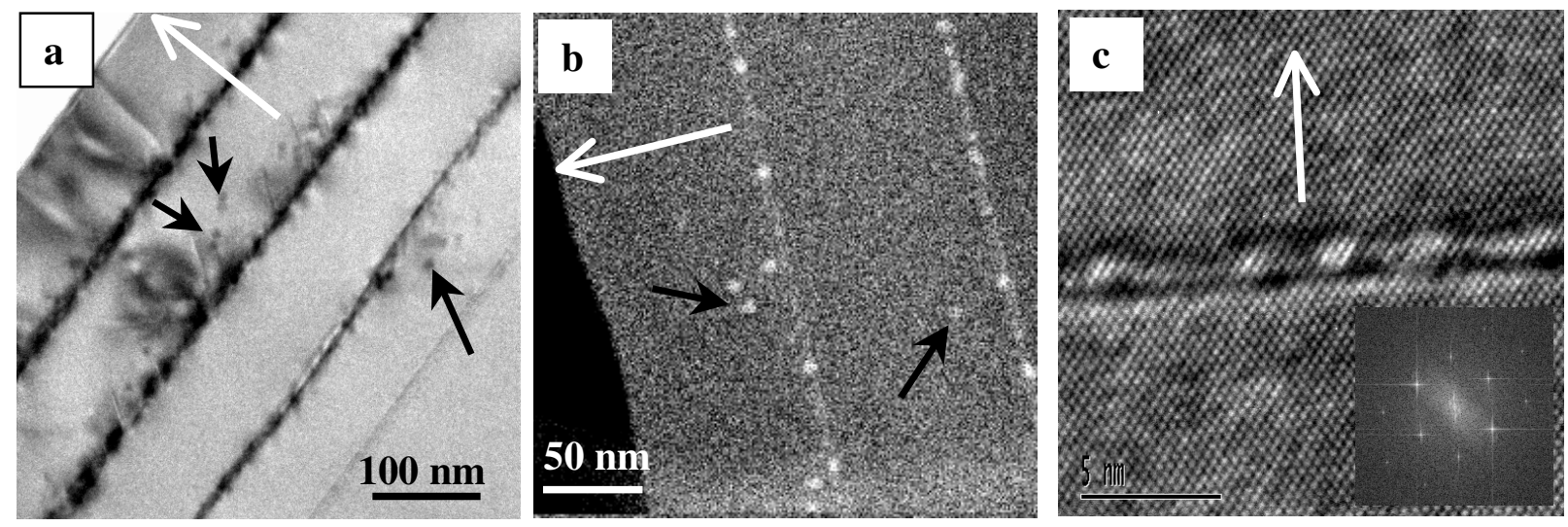

Figure 2. $\mathrm{Sn}_{\mathrm{x}} \mathrm{Si}_{1-\mathrm{x}} / \mathrm{Si}$ multilayer structures in [110] cross sections, the smaller black arrows points towards $\mathrm{Sn}$ precipitates that grew within the Si layers, the larger white arrows indicate growth directions; (a) CTEM overview, $\mathrm{x}$ = 0.1, ex-situ annealed; (b) Z-contrast STEM overview, $\mathrm{x}=0.1$, ex-situ annealed; (c) HRTEM image showing a perfectly pseudomorph $\mathrm{Sn}_{0.02} \mathrm{Si}_{0.98}$ layer as deposited, power spectrum as insert.

An interesting question is how these voids may have arisen in the first place. The answer to this question may be found in a mechanism analogously to that described in ref. [19]. According to that mechanism, when a freshly prepared $\mathrm{Si}$ surface is exposed to air at room temperature, voids of about $10 \mathrm{~nm}$ diameter and a number density of about $10^{10} \mathrm{~cm}^{-2}$ form spontaneously approximately $10 \mathrm{~nm}$ below the surface due to compressive strain that arises 
from the formation of $\mathrm{SiO}_{2}$ on the surface [19]. Temperature and growth rate modulated MBE deposited $\mathrm{Sn}_{\mathrm{x}} \mathrm{Si}_{1-\mathrm{x}}$ layers may also cause the formation of voids during the (low temperature) growth process since they also compress a freshly grown Si surface. The thermal cycling during temperature and growth rate modulated MBE [8,9] of multilayer structures ensures that there are many vacancies in the structure and this could allow preformed voids of any shape to grow and reach their equilibrium shape.
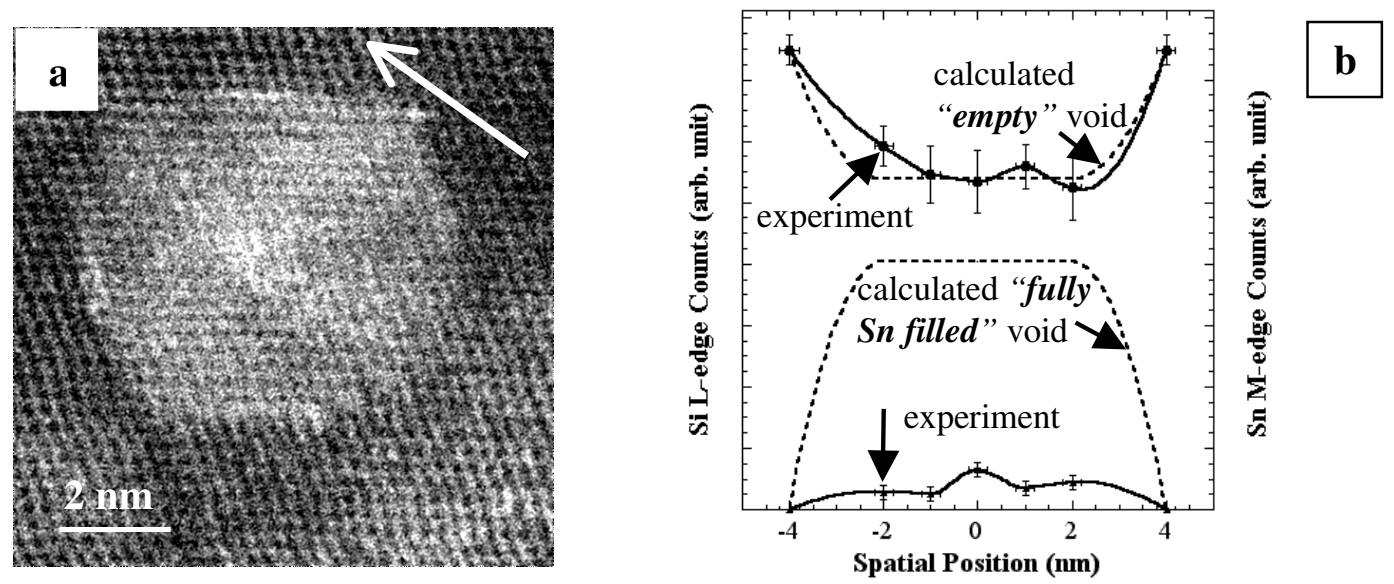

Figure 3. (a) [110] cross section Z-contrast STEM image of a void in Si that is lined by Sn on its interface with the Si matrix. The arrow represents the growth direction, $x=0.1$, ex-situ annealed. (b) Si L-edge counts and Sn M-edge counts at a series of spatial positions across a void (and along a line perpendicular to the growth direction) in Si that is partly filled with Sn. While the solid circles represent the experimental Si L-edge counts, the triangles represent the experimental Sn M-edge counts. Calculated Si L-edge and Sn M-edge counts are superimposed as dashed lines for both assumptions, that this void is either "empty" or "fully Sn filled". The close proximity of the experimental and calculated sets of Si L-edge counts and the large difference between the respective sets of Sn M-edge counts both indicate that the EELS data are due to a partially Sn filled void in Si. The calculations were performed for a tetrakaidecahedron with $\mathrm{A}=0.26$ and a specimen thickness of $33 \pm 3 \mathrm{~nm}$, as determined experimentally.
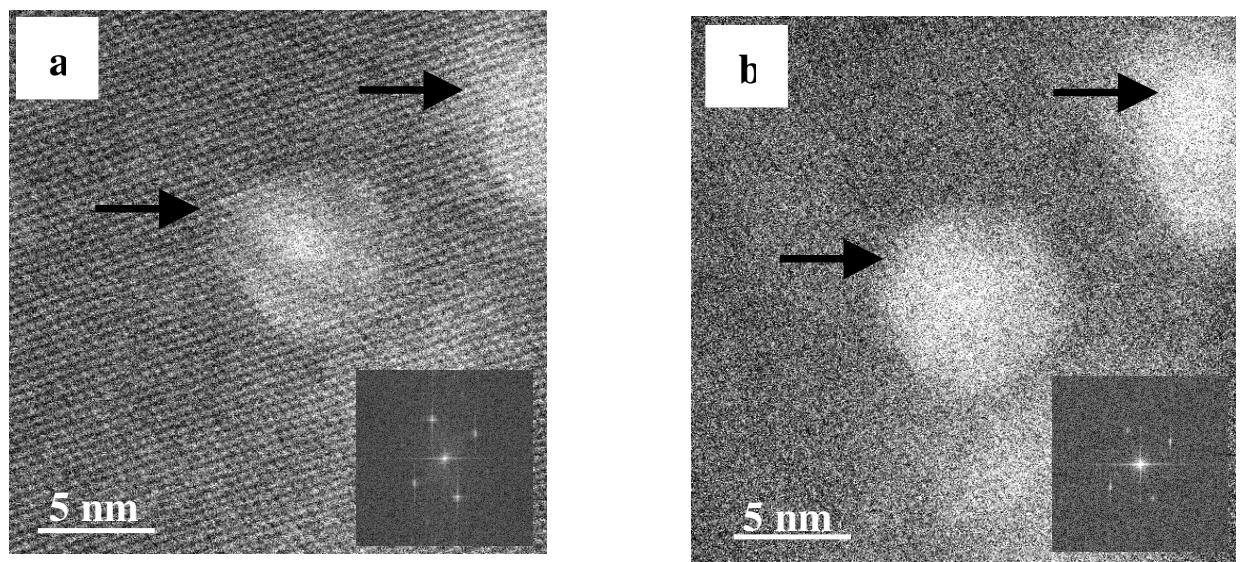

Figure 4. [110] cross section Z-contrast STEM images of $\alpha$-Sn QD precipitates, $x=0.05$, ex-situ annealed, power spectra as inserts; (a) Voids in Si that are partially filled with Sn, marked by arrows; (b) The same voids but significantly more filled with $\mathrm{Sn}$ as a result of a moderate additional thermal treatment (in situ) under the STEM.

Phase separation of $\mathrm{Sn}$ from a pseudomorph $\mathrm{Sn}_{\mathrm{x}} \mathrm{Si}_{1-\mathrm{x}}$ predecessor layer results in QD precipitates as well since the diamond structural prototype can be conserved. Fig. 5a shows an early stage of the formation of such a QD at the spatial position of a pseudomorph $\mathrm{Sn}_{0.1} \mathrm{Si}_{0.9}$ layer. When fully formed, these QDs are substitutional solid solutions of $\mathrm{Sn}$ in $\mathrm{Si}$ (i.e. $\mathrm{Sn}_{\mathrm{y}} \mathrm{Si}_{1-\mathrm{y}}$ ) with a higher $\mathrm{Sn}$ content than the pseudomorph $\mathrm{Sn}_{\mathrm{x}} \mathrm{Si}_{1-\mathrm{x}}$ predecessor layers (i.e. y $>\mathrm{x}$ ). We consider this mechanism as the second formation mechanism for endotaxially grown QDs in the $\mathrm{Sn} / \mathrm{Si}$ system. While we proposed this mechanism earlier on theoretical grounds [9,20], the Z-contrast STEM images, e.g. Fig. 5a, suggest that this phase separation starts with the formation of $\{111\} \mathrm{Sn}-\mathrm{Si}$ interfaces and preferential substitutional 
Sn-Si replacements in the areas around intersecting $\{111\}$ planes. Note that only for a very high Sn content, Fig. 5b, is a direct band gap predicted for $\mathrm{Sn}_{\mathrm{x}} \mathrm{Si}_{1-\mathrm{x}}$ alloys [3].

A shape transition with size of $\mathrm{Sn}$ rich precipitates that may be due to an increasing contribution of the elastic mismatch strain energy to the total energy of the QDs has been observed, Figs. 5b,c. While smaller $\alpha$-Sn and Sn rich $\mathrm{Sn}_{\mathrm{y}} \mathrm{Si}_{1-\mathrm{y}} \mathrm{QD}$ precipitates possess the typical tetrakaidecahedron shape, Figs. $1 \mathrm{~b}, 4 \mathrm{~b}$, and $5 \mathrm{~b}$, which probably results from the anisotropy of the interface energy density [16], a much larger precipitate had a shape that resembles more closely an octahedron, Fig. 5c. The shape of this large precipitate may results from the anisotropy of the elastic mismatch strain energy, i.e. the thermodynamics of misfitting precipitates in other words [15].
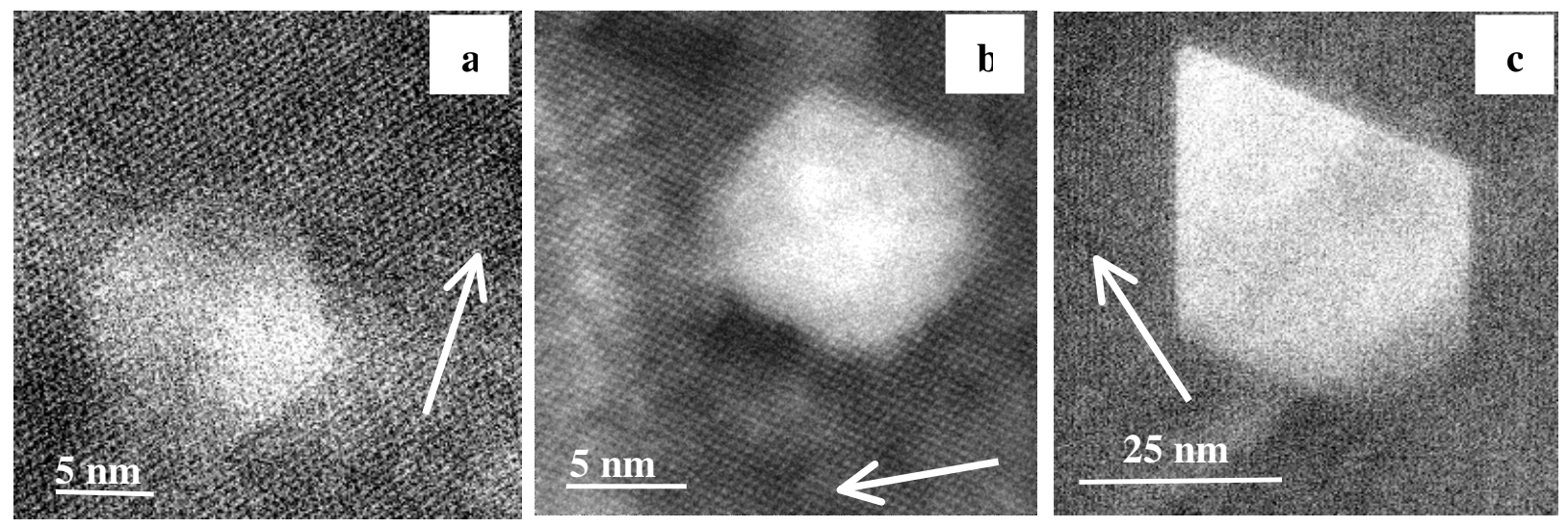

Figure 5. [110] cross section Z-contrast STEM images, $x=0.1$, ex-situ annealed, the arrows represent the respective growth directions; (a) Partially formed $\mathrm{Sn}_{\mathrm{y}} \mathrm{Si}_{1-\mathrm{y}}$ precipitate in $\mathrm{Si}$ with $\mathrm{y}>\mathrm{x}=0.1$, grown endotaxially by phase separation from a $\mathrm{Sn}_{0.1} \mathrm{Si}_{0.9}$ layer. Shape transition of $\mathrm{Sn}$ rich precipitates with size from (b) a tetrakaidecahedron to (c) essentially an octahedron.

Intermediately sized Sn rich QD precipitates possess tetrakaidecahedron shapes with smaller $\{001\}$ facets, i.e. smaller shape parameters suggesting a gradual transition to the shape of an octahedron $(\mathrm{A}=0)$ with increasing size. As the large precipitate in Fig. $5 \mathrm{c}$ is located partly at the predecessor substitutional $\mathrm{Sn}_{0.1} \mathrm{Si}_{0.9}$ solution layer and partly within the Si spacer layer, it may have formed by the simultaneous operation of both endotaxial growth mechanisms. The upper part of the QD may, therefore, consist of $\alpha$-Sn and the lower part of a substitutional $\mathrm{Sn}_{\mathrm{y}} \mathrm{Si}_{1-\mathrm{y}}$ solution with a high Sn content. This hypothesis is consistent with the contrast in Fig. $5 \mathrm{c}$ as there seems to be comparatively less $\mathrm{Sn}$ in the center of this precipitate that around its $\{111\} /(001)$ apex.

Finally, we would like to suggest that the employment of the void creation and subsequent filling mechanism (by with $\alpha$-Sn precipitate QDs grow endotaxially in $\mathrm{Si}$ ) may offer an opportunity to make progress in other QD systems such as endotaxially grown (In,As, $\mathrm{Si}$ ) QDs in $\mathrm{Si}$ [21]. With the equilibrium shape of the voids [13] may probably also be connected an equilibrium size so that QDs with a rather homogenous size distribution may be created by the employment of the newly proposed mechanism.

\section{SUMMAY AND CONCLUSIONS}

Two distinct mechanisms for the endotaxial growth of quantum dots in the $\mathrm{Sn} / \mathrm{Si}$ system have been observed. The first of these mechanisms is of a novel type and involves the creation of voids in the Si matrix and their subsequent filling with Sn atoms by diffusion. The second mechanism results from phase separation, as proposed earlier. While the QDs that result from the first mechanism consist of pure $\alpha-S n$, the quantum dots that result from the second mechanism are substitutional $\mathrm{Sn}_{\mathrm{y}} \mathrm{Si}_{1-\mathrm{y}}$ solutions with a high $\mathrm{Sn}$ content. Both of these mechanisms result in QDs which possess the diamond structure and the typical shape of a tetrakaidecahedron. Sn precipitates that are several times larger than the typical quantum dot possess an essentially octahedral shape.

\section{ACKNOWLEDGMENTS}

This research was supported by both a grant to PM by the Campus Research Board of the University of Illinois at Chicago and a grant to NDB by the National Science Foundation (DMR-9733895). The growth of $\mathrm{Sn}_{\mathrm{x}} \mathrm{Si}_{1-x} / \mathrm{Si}$ structures was supported by a National Science Foundation grant (ECS-0103543) to HAA. 


\section{REFERENCES}

[1] D. Bimberg, "Quantum dots: Paradigm changes in semiconductor physics", Semiconductors 33, 951-955 (1999).

[2] N.N. Ledentsov, V.M. Ustinov, V.A. Shchukin, P.S. Kop'ev, Zh.I. Alferov, and D. Bimberg, "Quantum dot heterostructures: fabrication, properties, lasers (Review)", Semiconductors 32, 343-365 (1998).

[3] R.A. Soref and C.H. Perry, "Predicted band gap of the new semiconductor SiGeSn", J. Appl. Phys. 69, 539-541 (1991).

[4] S.Y. Shiryaev, J. Lundsgaard Hansen, P. Kringhøj, and A.N. Larsen, "Pseudomorphic $\mathrm{Si}_{1-\mathrm{x}} \mathrm{Sn}_{\mathrm{x}}$ alloy films grown by molecular beam epitaxy on Si”, Appl. Phys. Lett. 67, 2287-2289 (1995).

[5] M.F. Flyn, J. Chevallier, J. Lundsgaard Hansen, and A. Nylandsted Larsen, "Relaxation of strained, epitaxial $\mathrm{Si}_{1-\mathrm{x}} \mathrm{Sn}_{\mathrm{x}} ”$, J. Vac. Sci. Technol. B 16, 1777-1785 (1998).

[6] M.F. Flyn, J. Chevallier, A.Nylandsted Larsen, R. Feidenhans'l, and M. Seibt, “ $\alpha-S n$ and $\beta$-Sn precipitated in annealed $\mathrm{Si}_{0.95} \mathrm{Sn}_{0.05}$ ", Phys. Rev. B 60, 5770-5777 (1999).

[7] C. Ridder, M. Fanciulli, A. Nylandsted Larsen, and G. Weyer, "Precipitation of Sn in metastable, pseudomorphic $\mathrm{Si}_{0.95} \mathrm{Sn}_{0.05}$ films grown by molecular beam epitaxy", Mater. Sci. Semicond. Processing 3, 251-255 (2000).

[8] K.S. Min and H.A. Atwater, "Ultrathin pseudomorphic $\mathrm{Sn} / \mathrm{Si}$ and $\mathrm{Sn}_{\mathrm{x}} \mathrm{S}_{1-\mathrm{x}} / \mathrm{Si}$ heterostructures", Appl. Phys. Lett. 72, 1884-1886 (1998).

[9] R. Ragan, K.S. Min, and H.A. Atwater, "Direct energy gap group IV semiconductor alloys and quantum dot arrays in $\mathrm{Sn}_{\mathrm{x}} \mathrm{Ge}_{1-\mathrm{x}} / \mathrm{Ge}$ and $\mathrm{Sn}_{\mathrm{x}} \mathrm{Si}_{1-\mathrm{x}} / \mathrm{Si}$ alloy systems", Mater. Sci. Engin. B 87, 204-213 (2001).

[10] P. Möck, Y. Lei, T. Topuria, N.D. Browning, R. Ragan, K.S. Min, and H.A. Atwater, "Structural and Morphological Transformations in Self-assembled Sn Quantum Dots in Si Matrix", Proc. 2003 Nanotechnology Conference and Trade Show, Vol. 3, 74-77, 2003.

[11] P. Möck, T. Topuria, N.D. Browning, M. Dobrowolska, S. Lee, J.K. Furdyna, G.R. Booker, N.J. Mason, and R.J. Nicholas, "Internal self-ordering in $\mathrm{In}(\mathrm{Sb}, \mathrm{As})$, (In, $\mathrm{Ga}) \mathrm{Sb}$ and $(\mathrm{Cd}, \mathrm{Mn}, \mathrm{Zn}) \mathrm{Se}$ nano-agglomerates/quantum dots", Appl. Phys. Lett. 79, 946-948 (2001).

[12] P. Möck, Y. Lei, T. Topuria, N.D. Browning, R. Ragan, K.S. Min, and H.A. Atwater, "Structural transformations in self-assembled semiconductor quantum dots as inferred by transmission electron microscopy", Physical Chemistry of Interfaces and Nanomaterials, Jin Z. Zhang, Zhong L. Wang, Eds. Proc. of SPIE Vol. 4807, 71-82 (2002).

[13] D.J. Eaglesham, A.E. White, L.C. Feldman, N. Moriya, and D.C. Jacobson, "Equilibrium Shape of Si", Phys. Rev. Lett. 70, 1643-1646 (1993).

[14] D.A. Porter and K.E. Easterling, Phase Transformations in Metals and Alloys, Chapman \& Hall, London, New York, 1992.

[15] W.C. Johnson, "Influence of Elastic Stress on Phase Transformations", in: Lectures on the Theory of Phase Transformations, 2nd Edition, Ed. H.I. Aaronson, The Minerals, Metals \& Materials Society, Warrendale, 2001.

[16] Neumann's symmetry principle, $\frac{4}{m} \overline{3} \frac{2}{m} \cap \frac{4}{m} \overline{3} \frac{2}{m}=\frac{4}{m} \overline{3} \frac{2}{m}$, yields as the polyhedra that are consistent with the point symmetry group of the interface energy density a tetrakaidecahedron, an octahedron, and a cube. Out of these polyhedra, the tetrakaidecahedron possesses the least symmetry as it has a shape parameter. The shapes of small misfitting precipitates are usually dominated by the anisotropy of the interface energy density [15] and the smallest Sn precipitates should, therefore, possess the shape of a tetrakaidecahedron. Since the lattice mismatch stress (pressure) is essentially hydrostatic, i.e. isotropic, Curie's symmetry principle, $\infty \infty \infty$ $\cap \frac{4}{m} \overline{3} \frac{2}{m}=\frac{4}{m} \overline{3} \frac{2}{m}$, yields no influence of the misfit stress field on the anisotropy of the interface energy density.

[17] Y. Lei, "Atomic scale analysis of semiconductor quantum dots by scanning transmission electron microscopy", PhD thesis, 2003, University of Illinois at Chicago.

[18] Y. Lei, P. Möck, T. Topuria, N.D. Browning, R. Ragan, K.S. Min, and H.A. Atwater, "Void Mediated Formation of Sn Quantum Dots in a Si Matrix", Appl. Phys. Lett., accepted.

[19] S.H. Lin, I. Mack, N. Pongkrapan, and P. Fraundorf, "Ten-nanometer surface intrusions in room temperature silicon", Electrochem. \& Solid State Lett. 5, G83-G85 (2002), con-mat/0110393.

[20] K.S. Min, "Synthesis and Properties of Light-Emitting Si-Based Nanostructures", PhD thesis, 1999, California Institute of Technology.

[21] N.D. Zakharov, P. Werner, U. Gösele, R. Heitz, D. Bimberg, N.N. Ledentsov, V.M. Ustinov, B.V. Volovik, Zh. I. Alferov, N.K. Poluakov, V.N. Petrov, V.A. Egorov, and G.E. Cirlin, "Structure and optical properties of Si/InAs/Si layers grown by molecular beam epitaxy on Si substrate", Appl. Phys. Lett. 76, 2677-2679 (2000). 